\title{
Analysis of 3D stacked fully functional CMOS Active Pixel Sensor detectors
}

\author{
D. Passeri, ${ }^{a, 1}$ L. Servoli ${ }^{b}$ and S. Meroli ${ }^{b}$ \\ ${ }^{a}$ University of Perugia, \\ via G. Duranti 93, 06125 Perugia, Italy \\ ${ }^{b}$ Istituto Nazionale di Fisica Nucleare, \\ via A. Pascoli 1, 06123 Perugia, Italy \\ E-mail: daniele.passeri@diei.unipg. it
}

ABSTRACT: The IC technology trend is to move from 3D flexible configurations (package on package, stacked dies) to real 3D ICs. This is mainly due to $i$ ) the increased electrical performances and ii) the cost of 3D integration which may be cheaper than to keep shrinking 2D circuits. Perspective advantages for particle tracking and vertex detectors applications in High Energy Physics can be envisaged: in this work, we will focus on the capabilities of the state-of-the-art vertical scale integration technologies, allowing for the fabrication of very compact, fully functional, multiple layers CMOS Active Pixel Sensor (APS) detectors. The main idea is to exploit the features of the 3D technologies for the fabrication of a "stack" of very thin and precisely aligned CMOS APS layers, leading to a single, integrated, multi-layers pixel sensor. The adoption of multiple-layers single detectors can dramatically reduce the mass of conventional, separated detectors (thus reducing multiple scattering issues), at the same time allowing for very precise measurements of particle trajectory and momentum. As a proof of concept, an extensive device and circuit simulation activity has been carried out, aiming at evaluate the suitability of such a kind of CMOS active pixel layers for particle tracking purposes.

KEYWORDS: Particle tracking detectors, Detector modelling and simulations II (electric fields, charge transport, multiplication and induction, pulse formation, electron emission, etc)

\footnotetext{
${ }^{1}$ Corresponding author.
} 


\section{Contents}

1 Introduction 1

2 The simulated structures 2

3 Impact point and incidence angle reconstruction 2

4 Design options evaluation 4

4.1 Adjacent layers coupling options 5

$\begin{array}{lll}4.2 & \text { Pitch analyses } & 6\end{array}$

5 Conclusions 6

\section{Introduction}

The future generation of High Energy Physics (HEP) experiments will demand stringent requirements for tracking systems, in particular in terms of low material budget and read-out speed. Within this framework, the advance of the microelectronics technology already fostered the adoption of monolithic detection systems based on CMOS Active Pixel Sensors (APS) structures. The suitability of such a class of sensors for Ionizing Particle detection has been already assessed [1,2]. Along with the progress of the microelectronics fabrication technology, a new generation of silicon detectors can be envisaged. Actually, the IC technology trend is to move from 3D flexible configurations (package on package, stacked dies) to real 3D ICs. This is mainly due to $i$ ) the increased electrical performance which can be obtained, and ii) to the cost of 3D integration which may be cheaper than to keep shrinking 2D circuits. Perspective advantages for particle tracking and vertex detectors applications in HEP will be enabled from the separation of the sensor layer from the (analog) read-out electronics layers, as well as from A/D conversion layers. This will allow, e.g., for an increased fill-factor of the sensor and increased performance due to the lower impact of the interconnection length.

In this work, we will focus on the capabilities of the state-of-the-art vertical scale integration technologies, aiming at the fabrication of very compact, fully functional, multiple layers CMOS APS detectors. The main idea is to exploit the features of the 3D technologies for the integration of a "stack" of very thin and precisely aligned CMOS APS layers, leading to a single, monolithic, multi-layers pixel sensor. The adoption of multiple-layer all-in-one detectors can dramatically reduce the mass of a conventional, separated detectors (thus reducing multiple scattering issues), at the same time allowing for very precise measurements of a particle trajectory and momentum (e.g. by using a couple of multiple-layer single detectors spatially separated). 


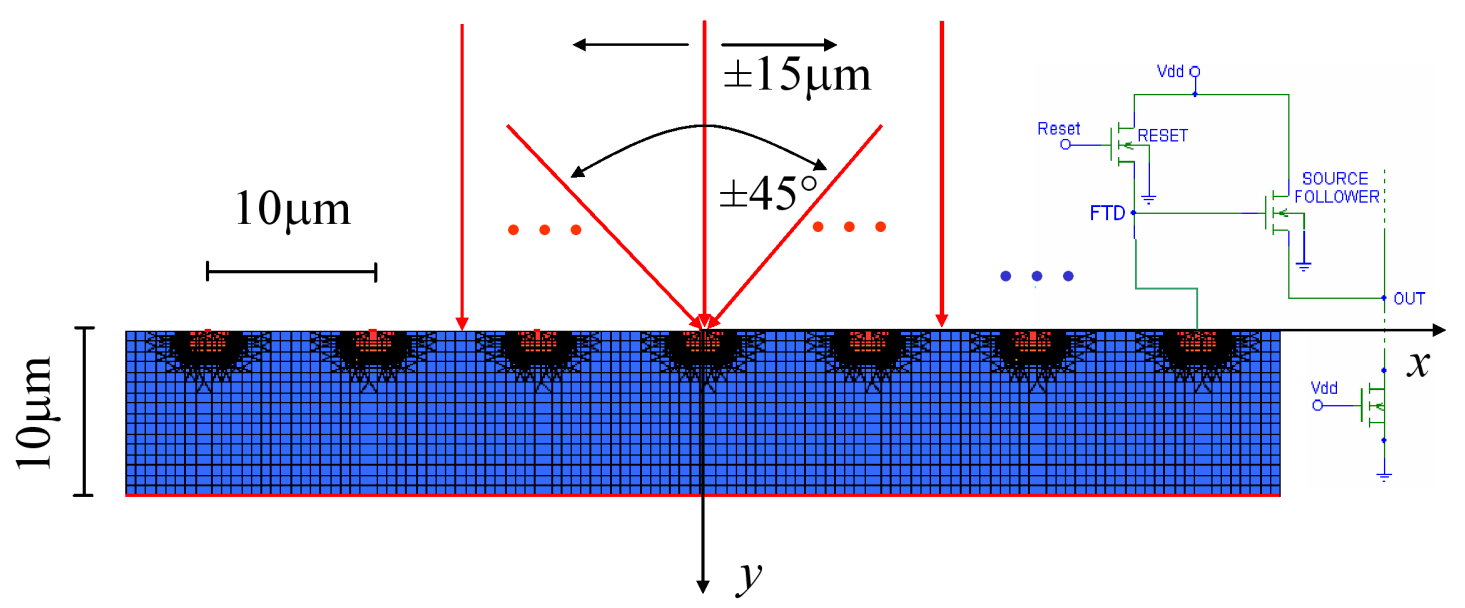

Figure 1. 2D cross-section of a CMOS Active Pixel Sensor sub-array (7 pixels).

\section{The simulated structures}

As a proof of concept, an extensive simulation activity has been carried out, starting from a simple 2D section of a 7-pixels sub-array. Mixed-mode (namely device-circuit) simulations have been carried out: each pixel sensitive element (photodiode) has been coupled to the three transistor circuit APS scheme (figure 1). A Minimum Ionizing Particle stimuli response has been therefore simulated, considering the distribution of the charge for an impinging particle. The combinations of several incidence points ranging from -15 micrometers to +15 micrometers with respect to the central pixel and incidence angles ranging from $-45^{\circ}$ to $+45^{\circ}$ with respect to the normal incidence have been considered. The voltage responses of each pixel as a function of both position and angle have been calculated by distributing a fixed amount of electron/holes pairs along the particle track. In particular, $80 \mathrm{e} / \mathrm{h} /$ micron have been considered as a starting point of a transient (time-varying) analysis. All the results were obtained by considering both charge drift and diffusion components, for a typical CMOS substrate doping concentration and under biasing conditions, and the load effect of reset and source-follower MOSFETs. As an example, in figure 2 are reported the voltage drops of the central pixel as a function of the incidence point and impact position. In particular, the track "A" (see the inset) refers to a normal incidence (angle $=0$ ) and central impact position $(\mathrm{x}=0)$, whereas track "B" refers to a angled incidence (angle $=45)$ and sided impact position $(\mathrm{x}=-5 \mu \mathrm{m})$. The highest response related to track $\mathrm{B}$ is due to the longest particle path along the silicon substrate and therefore to a bigger amount of generated electron/holes pairs.

Multiple layer responses (up to 4 layers) have been subsequently simulated. All layers have been considered as electrically independent and for each layer all pixel responses have reported (figure 3).

\section{Impact point and incidence angle reconstruction}

In order to evaluate the suitability of having multiple spatially close measuring layers the impact point and the incidence angle reconstruction have been performed, starting from the voltage responses at the pixel outputs. In particular, we considered a cluster signal, featuring three pixel 


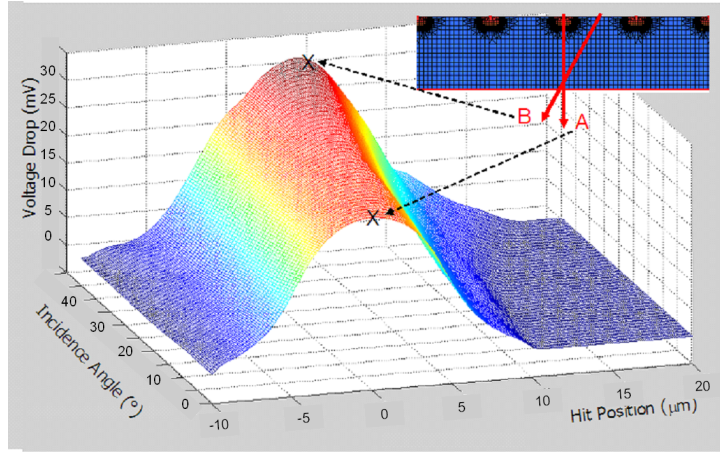

Figure 2. Single layer structure: central pixel response (voltage drop) to a particle hit, as a function of the impact point and incidence angle.

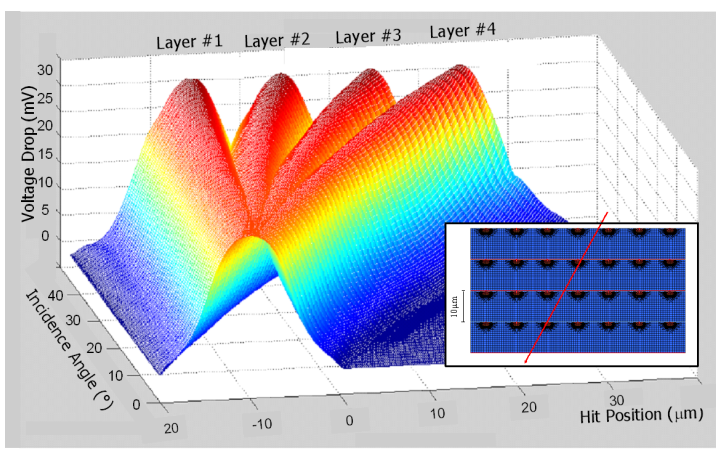

Figure 3. Four layer structure: central pixel responses to a particle hit, as a function of the impact point and incidence angle.

responses, namely the seed pixel (the pixel with highest response to a particle crossing) and the two neighboring ones. Then, we considered the effect of the (dominant) kTC equivalent noise contribution, as measured from available APS sensors (in the order of $1.0 \mathrm{mV}$ ) [3].

The equivalent noise voltage was therefore added to the voltage response of each pixel using a Gaussian distribution. The hit position and angle were therefore reconstructed from the voltage response of each pixel involved in the cluster, by a weighted average of the voltage signals between pixels pertaining to the same layer and by a linear fitting of the information obtained from different layers (namely, we considered as straight line the trajectory of the particle inside the detector substrate). As an example, the standard deviation of the distribution of the impact point and incidence angle reconstruction for a given trajectory are reported in figures 4, 5 and figures 6, 7, respectively. Such a findings can be used as a measure of the reconstruction error. In particular, in figure 4 is reported the error on the impact point reconstruction when considering the information coming from only two layers, whereas the same result when considering three layers is reported in figure 5. In this case, a small improvement of the accuracy can be appreciated. On the other hand, a significant improvement on the incidence angle reconstruction accuracy can be appreciated, moving from two layers structure (figure 6) to three layers structure (figure 7).

These results can be extended to different impact points and incidence angles. A comprehensive analysis of the four layers sensor structure is reported is figures 8 and 9 , where the above discussed results have been summarized by considering different particle trajectories. In particular, we considered an impact point spanning from a left half-pitch with respect to the central pixel to a right half-pixel and incidence angle ranging from $0^{\circ}$ to $45^{\circ}$ with respect to the normal incidence. In this case it is possible to point out that the effect of the noise is higher for hits that occur very close to the central pixel and with normal incidence. Actually, the amount of the cluster charge is minimum in this situation, since only the seed pixel contributes to the cluster charge (mono-pixel cluster) and the particle path along the substrate is the shortest. However, the overall performance improvement when moving from two to three layers is evident as well, in particular in terms of angle reconstruction. A further increase of the layer numbers (from three to four layers) will result in only small improvement of the precision. 


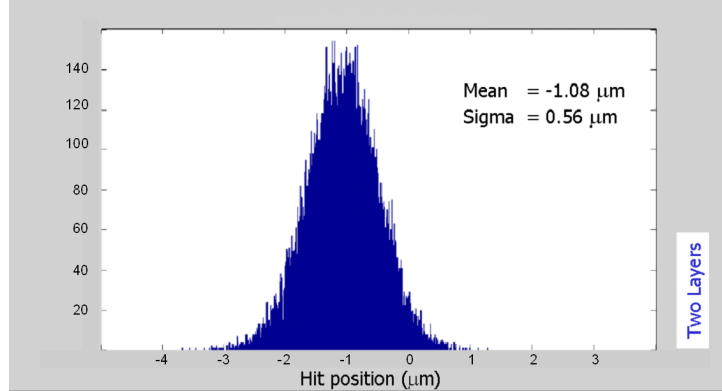

Figure 4. Hit position error reconstruction estimation: two layers structure $\left(\mathrm{x}=0 \mu \mathrm{m}\right.$, angle $\left.=20^{\circ}\right)$.

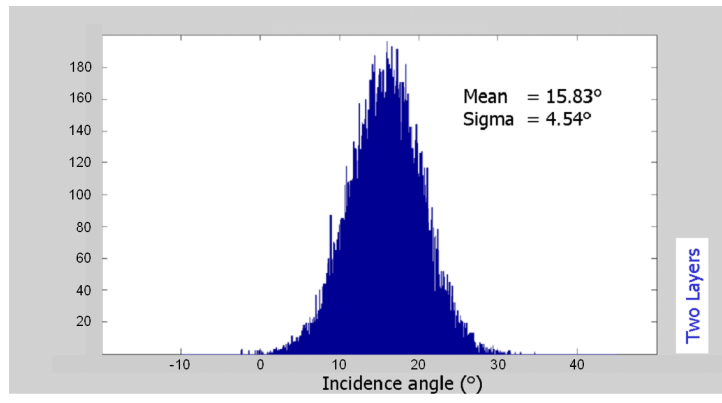

Figure 6. Incidence angle error reconstruction estimation: two layers structure $(\mathrm{x}=0 \mu \mathrm{m}$, angle $=20^{\circ}$ ).

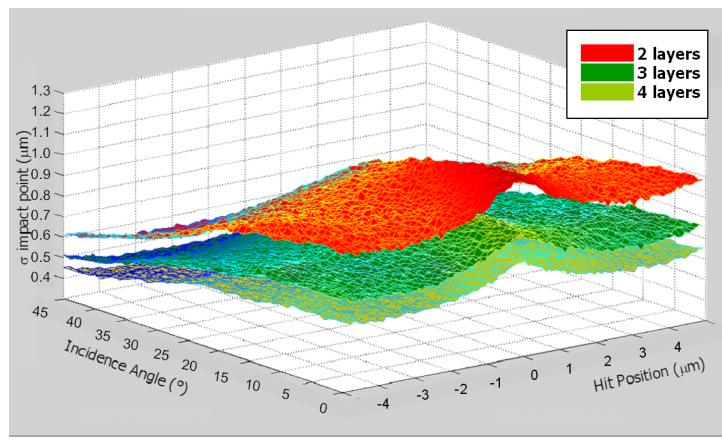

Figure 8. Standard deviation of the impact point calculation as a function of the incidence angle and hit position.

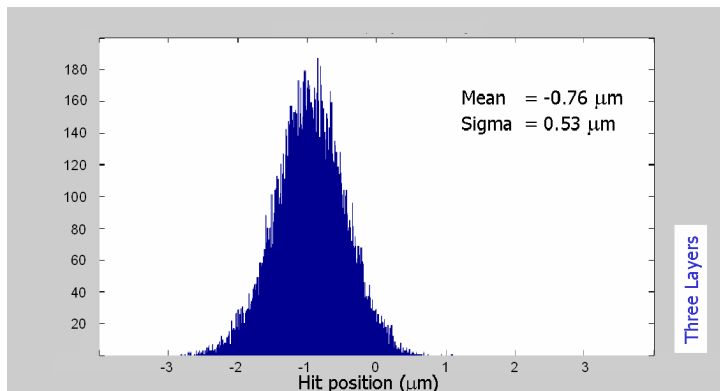

Figure 5. Hit position error reconstruction estimation: three layers structure $\left(\mathrm{x}=0 \mu \mathrm{m}\right.$, angle $\left.=20^{\circ}\right)$.

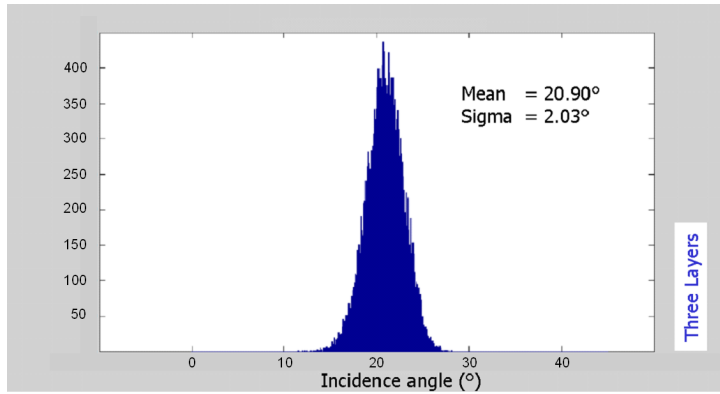

Figure 7. Incidence angle error reconstruction estimation: three layers structure $(\mathrm{x}=0 \mu \mathrm{m}$, angle $=$ $\left.20^{\circ}\right)$.

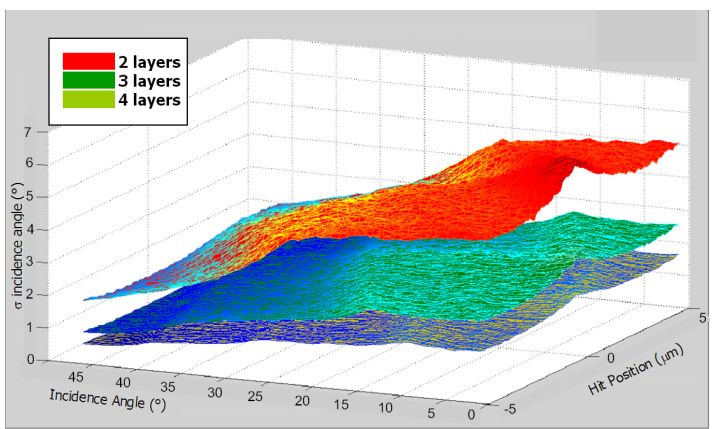

Figure 9. Standard deviation of the incidence angle calculation as a function of the incidence angle and hit position.

\section{Design options evaluation}

Once the simulation models and schemes are assessed, TCAD tools can be proficiently used as predictive mean in order to check the suitability of different VLSI design options, aiming at the optimization of the detector geometry for the specific task at hand. 

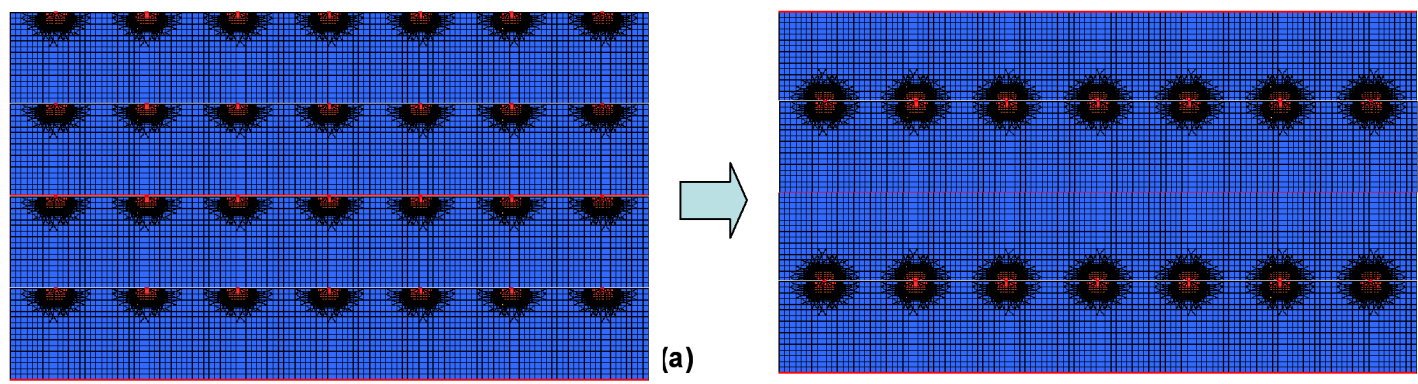

(b)

Figure 10. Possible layouts of multiple stacked layers: four layers front to back coupled (a) and two couples of layers front to front coupled, and then (back to back) joined.

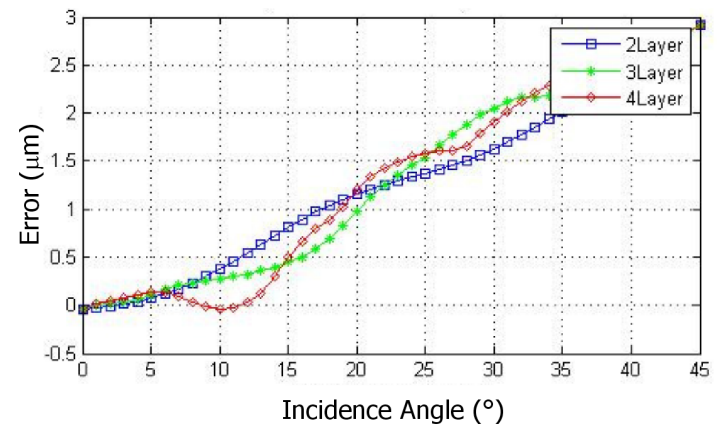

Figure 11. Impact point reconstruction error for a central hit trajectory (impact point coordinate $\mathrm{x}=$ $0 \mu \mathrm{m}$ ), for a front to back coupled layers (scheme (a) of figure 10 above).

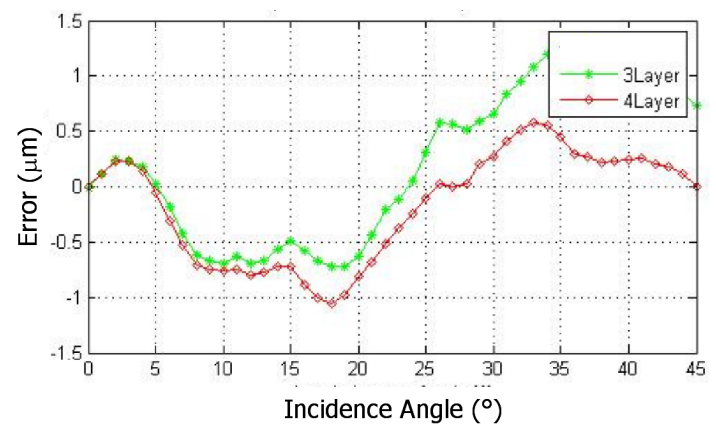

Figure 12. Impact point reconstruction error for a central hit trajectory (impact point coordinate $\mathrm{x}=0 \mu \mathrm{m}$ ), for a front to front coupled layers as (scheme (b) of figure 10 above).

\subsection{Adjacent layers coupling options}

All the previous results have been obtained by considering equally vertically spaced layer, with front to back coupling (see the inset in figure 3). This is only a tentative layout for a multiplelayer detectors, and this is strongly technology dependent. Within this framework, a number of options have been explored as well. For instance, by considering only two layers, no significant performance difference can be appreciate from front to back and front to front coupling, whereas an interesting layout can be obtained by combining two front to front couples in a back to back sandwich (figure 10). In this case, the higher precision of the two upper and lower front to front coupled layers, along with the increased distance between the measurement points, will result in a smaller error on the impact point estimation, in particular for tilted trajectories, with respect to the previously considered four layer back to front coupled structure, even if the number of points which can be used for the track reconstruction is lower (figures 11 and 12). On the other hand, it will possible to find a trade-off between a track reconstruction scheme based upon several measurements, each one featuring a relatively large error and a track reconstruction with fewer measurements (the two layers front to back coupling will essentially result in a single layer measurement with lower error) but featuring higher accuracy. 


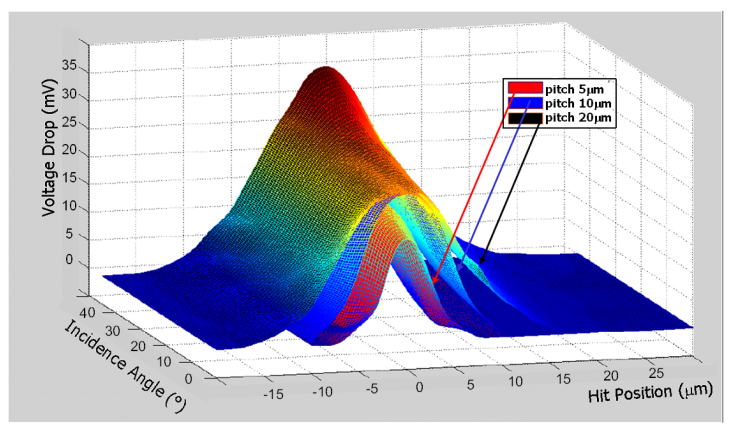

Figure 13. Hold-on of the responses of the central pixel for single layer structures featuring pitches of 5, 10 and 20 micrometers, respectively.

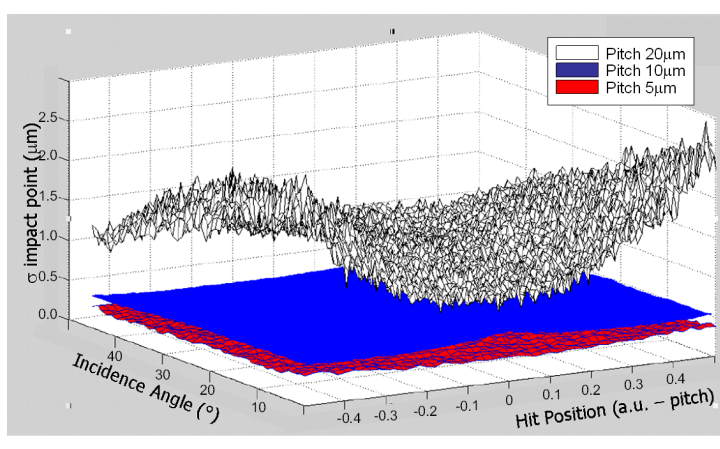

Figure 14. errors on the impact point reconstruction for the 5, 10 and 20 micrometers pitch structures.

\subsection{Pitch analyses}

Since the layer depth is typically a technology constraint (depending on the vias formation procedure) different configurations in terms of sensitive area dimensions and pitch can be investigated, in order to maximize the efficiency and the spatial resolution of vertex detectors for High Energy Physics applications. As an example, assuming a given dimension of the sensitive area (in this case $2 \times 2$ squared micrometers), the impact of different pitches on the charge collection properties have been checked. To this purpose, in figure 13 the hold-on of responses of the central pixel of the same structure illustrated in figure 1 (i.e. a single layer detector) have been reported, for pitches of 5, 10 and 20 micrometers, respectively. The smallest signal obtained with the smallest pitch configuration is due to the charge sharing between very closely spaced pixel (which results in a smaller amount of charge collected by the central pixel), whereas for wider structures most of the generated charge is collected by the seed pixel (i.e. the closest pixel to the particle trajectory). The analyses were therefore extended to evaluate the effect of multiple layer structures as well. In figure 14 are reported the errors on the impact point reconstruction, as previously defined, namely in terms of standard deviation of the distribution for the 5, 10 and 20 micrometers pitch structures. The effect of the pixel "spacing" is clearly visible: for a 20 micrometers pitch and for particle crossing in between pixels, the charge loss (due do the recombination effects, since the collecting regions are relatively far away from the generation point) is significant, and therefore a bigger effect of the noise (which is compared to a smaller signal) can be experienced, thus preventing the adoption of very spaced pixel detector.

\section{Conclusions}

Vertical scale integrated circuits (3D ICs) have been already assessed as viable and promising option for the fabrication of microlectronics devices and circuits. This will enable new perspective for CMOS Active Pixel Sensors, which can be proficiently adopted in High Energy Physics experiments. In this work, we discussed the suitability of having a all-in-one detector featuring multiple stacked APS CMOS layers for particle tracking applications. To this purpose, comprehensive device/circuit simulations have been performed, demonstrating the advantaged of having two/three stacked (closed, very precisely aligned) fully-functional pixel layers in terms of track impact point 
and angle reconstruction precision. This will actually allow for particle momentum measurement with a single detector, as well as the fabrication of very low material detector, thus reducing the impact of multiple scattering issues in new generation vertex detectors.

\section{References}

[1] H.S. Matis et al., Charged Particle Detection Using a CMOS Active Pixel Sensor, IEEE Trans. Nucl. Sci. 50 (2003) 1020.

[2] L. Servoli et al., Test of a MAPS realized in standard non-epitaxial CMOS 0.18 $\mu$ m technology, Nucl. Instrum. Meth. A 581 (2007) 335.

[3] D. Passeri et al., Characterization of Active Pixel Sensors fabricated in CMOS 0.18 $\mu$ m technology, Nucl. Instrum. Meth. A 582 (2007) 871. 